\title{
ZeitschriftfürangewandteChemie.
}

\author{
Organ des Vereins deutscher Chemiker.
}

\author{
XX. Jahrgang.
}

Heft 42.

18. Oktober 1907.

Alleinige Annahme von Inseraten bei August Scherl, G. m. b. H., Berlin SW 68, Zimmerstr. $37 / 41$ und Daube \& Co., G. m. b. H., Berlin SW 19, Jerusalemerstr. 53/54

sowie in deren Filialen: Bremen, Obernstr. 16. Breelau, Schweidnitzerstr, 11. Chemnitz Sa., Marktgákehen 3. Dresden, Seestr. 1. Elberfeld, Herzogstr. 38. Frankfurt a. Y., Kaiserstr. 10. Halle a. G., Große Stein. 11 Hamburg, Alter Wall 76. Hannover, Georgstr. 39. Kaesel, Obere Königstr. 27. K81n a. Rh. To Nürberg, Kaiserstr, Ecke Fleischbrtuke. Etraßburg 1. E., Gießhausgasse 18/22, Stuttgart, Königstr. 11, I. Wien I, Graben 28. Wtirzburg, Franziskanergasse 51/g. Zilrioh, Bahnhofstr. 89.

Der Insertionspreis betrugt pro $\mathrm{mm}$ Höhe bei $15 \mathrm{~mm}$ Breite (8 gespalten) 15 Pfennige, auf den beiden auferen Umschlagseiten 20 Pfennige. Bei Wiederholungen tritt entsprechender Rabatt ein. Beilagen werden pro 1000 Stuck mit 10.50 M ftir 5 Gramm Gewicht berechnet; für schwere Beilagen tritt besondere Vereinbarung ein.

I N H A I T:

F. Raschig: Entgegnung an G. Lunge und E. Berl 1809

D. Binder: Die erste bekannte Kohlenoxydvergiftung 1811

O. A s c han: Uuber den Vorlauf des finlandischen Terpentinöles (Kienöles) 1811. Gerichtliche Entscheidungen in Patentsachen 1816.

\author{
Referate: \\ Kautschuk, Guttapercha 1818; - Bleicherei, Färberei und Zeugdruck 1819.
}

Wirtschaftlich-gewerblicher Teil:

Tagesgeschichtliche und Handelsrundschau: Zur Kupferkrise 1827; - Amerika; - Zuckererzeugung aus Holzkohle; - Boston; - Kupferfunde in Jamaika; - Rio de Janairo; - China 1828; - Japan; - Krisis unter den Chininproduzenten; - Britisch.Indien; - Das neue englische Patentgesetz und die chemische Industrie; - England; Bulgarien 1829; - Paris; - Belgien; - Das Berg. und Hüttenwesen in Bosnien und der Herzegowina im Jahre 1906; - Wien; - Budapest; - Deutschland 1830; - Aus der Zementindustrie; - Aluminiumpreise; - Handelsabkommen mit England; - Verband der deutschen Flaschenfabrikanten; - Berlin ; - Danzig; - Hamburg 1881; Posen; - Stuttgart; - Handelsnotizen 1832; - Dividenden; - Aus anderen Vereinen und Versammlungen: 14. Internationaler Kongreß für Hygiene und Demographie zu Berlin 1883; - Verband von Nahrungsmittelinteressenten; nasstellung für pharmazeutischen Bedarf; - Berliner Bezirksverein deutscher Ingenieure; - Österreichische PharAusstellung für pharmazeutisehen Bedarf; - Berliner Bezirksverein deutscher Ingenieure; - Osterreichische Phar-
mazeutische Gesellschaft; - Personal- und Hochschulnachrichten; - Post 1835; - Eingelaufene Bücher; - Bücherbesprechungen 1836; - Patentlisten 1839.

Verein deutscher Chemiker:

Ausdehnung der Infektionsklausel auf die Chemiker; - Bezirksverein Hannover 1840.

\section{Entgegnung an G. Lunge und E. Berl.}

Von F. RAschig-Ludwigshafen a. Rh.

(Eingegang. d. 30./9. 1907).

Meine Antwort auf die neuesten Mitteilungen von Lunge und Berli) kann sehr kurz gehalten werden; denn da sie den weitaus größten Teil meiner Ausführungen mit Stillschweigen übergehen und diese mit ihrer Charakterisierung als ,,spöttisch und unsachlich" doch nicht aus der Welt zu schaffen sind, so ist das, was sie heute vorbringen, selbst wenn es richtig wäre, ohne Einfluß auf die Beweiskraft meiner Darlegungen.

Leider ist es aber nicht einmal richtig; denn was ich über die Erscheinungen gesagt habe, die eintreten, wenn man Stickstoffdioxyd im Sauerstoffstrom verflüchtigt und das Gasgemisch in Natronlauge oder in konz. Schwefelsäure auffängt, daß es sich nämlich in ersterer genau als $\mathrm{NO}_{2}$, zu einem molekularen Gemisch von Nitrat und Nitrit, und in letzterer zu einem Gemisch, in dem die salpetrige Säure gegenüber der Salpetersäure etwas vorwiegt,

1) Diese Z. 20, 1713 (1907). auflöst, das halte ich nach wie vor fest. Ich weiß auch nicht, wie $\mathrm{L}$ u n ge und B e r l auf den Gedanken kommen konnten, ich hätte keine neuen Versuche in dieser Hinsicht angestellt. Das Gegenteil ist der Fall; es sind Hunderte von derartigen Versuchen gemacht worden, und $n \mathrm{i}$ e $\mathrm{m}$ a $1 \mathrm{~s}$ ist in Natronlauge mehr Nitrat als Nitrit gefunden worden. Aber ich kann nicht jeden Versuch, den ich anstelle, ausführlich beschreiben und drucken lassen; sonst müßte ich dicke Bücher verfassen, und das ist nun einmal nicht nach meinem Geschmack.

$\mathrm{L}$ u $\mathrm{n}$ g e und B e r 1 verschweigen auch, da B ich ihnen nachgewiesen habe ${ }^{2}$ ), wie sie früher dasselbe gefunden haben wie ich, in Natronlauge genau gleich viel Nitrat und Nitrit, in Schwefelsäure weniger Salpetersäure und mehr salpetrige Säure. Wollte i $c h$, wie $s$ i e es $m$ i r gegenüber tun, Zweifel an der wahrheitsgetreuen. Beschreibung i h r e r Versuche ausdrücken, so könnte ich hier a u $\mathrm{c} h$ sagen: ,Eine oder die andere dieser Angaben muB doch falsch sein". Ich gehe aber nicht so weit und zweifle nicht

2) Diese Z. 20, 711 (1907); Chem. Ztg. 28, 1243 (1904). 
an der Richtigkeit ihrer Angaben und Zahlen; aber ich zweifle daran, daß ihre Versuche so angestellt sind, daß nicht Fehlerquellen von ihnen unbekannter Art das Resultat getrübt haben. Ich fordere sie daher hiermit öffentlich auf, mir einen dritten Ort $\mathrm{zu}$ bestimmen, an dem mir vor einem beliebigen unparteiischen Sachverständigen Gelegenheit gegeben wird, die Richtigkeit meiner Angaben durch den Versuch zu beweisen; und ich zweifle nicht, daß auch $\mathrm{L}$ u $\mathrm{nge}$ und $\mathrm{B}$ er $\mathrm{l}$ bei dieser Gelegenheit auf gleiche Art für ihre Ergebnisse eintreten werden. Da m a $B$ sich dann herausstellen, wer Recht hat; und bis das geschehen ist, wird die Wissenschaft den Schluß am Ende des ersten Abschnittes, ,daß die anders lautenden Behauptungen $\mathrm{R}$ a s $\mathrm{ch}$ ig s endgültig als durchaus unrichtig anzusehen sind", als Ansicht e i n e r Seite auffassen müssen.

Der Abschnitt II rennt mit dem mathematischen Nachweis, daß bei der Oxydation des Stickoxyds durch Luft kein Stickstofftrioxyd entstehe, offene Türen ein. Denn über diese, früher von mir gehegte Anschauung bin ich ja längst hinweggekommen und habe nachgewiesen, daß die erste Oxydation schnell zum Isostickstofftetroxyd führt

$$
2 \mathrm{NO}+\mathrm{O}_{2}=\mathrm{N}_{2} \mathrm{O}_{4}
$$

und die zweite von da langsam zum Isostickstoffpentoxyd

$$
2 \mathrm{~N}_{2} \mathrm{O}_{4}+\mathrm{O}_{2}=2 \mathrm{~N}_{2} \mathrm{O}_{5} \text {. }
$$

Beides sind trimolekulare Reaktionen; und wenn wirklich - was ich nicht beurteilen kann - die mathematische Entwicklung von $\mathrm{L}$ unge und Berl dadurch, daß sie den Sauerstoffgehalt der Luft jetzt richtig ansetzen, nur noch besser geworden ist, als früher, so beweist sie doch nicht mehr, als daß der T e i 1 der zweiten Hälfte der Stickoxydoxydation, den sie allein ihrer Rechnung zugrunde legen, in einer trimolekularen Reaktion verläuft. Das stimmt mit meiner Auffassung des Vorganges so gut wie mit der von $\mathrm{L}$ u $\mathrm{n}$ ge und $\mathrm{B}$ er $\mathrm{l}$.

Auch mit der erneuerten graphischen Darstellung kommen sie nicht weiter. Unangenehm berührt von meinem Vorwurf, sie hätten die Kurve absichtlich verzerrt aufgetragen, erklären sie jetzt, sie wollten die Kurve (A) in der von mir bevorzugten Weise bringen. Leider aber $t \mathbf{u} \mathbf{n}$ sie es nicht, sondern wenden zum zweiten Male einen anderen Maßstab an, wie ich, so daß man den Knick wiederum nicht sieht. Hätten sie wirklich die Kurve so gebracht, wie ich es bevorzugt habe, so wären sie auch ganz von selbst zu meinen Bildern ${ }^{3}$ ) gekommen, die den Knick ganz deutlich zeigen. Und auf Grund einer solchen Beweisführung, die im Bild nicht hält, was sie im Text verspricht, wird dann der End. schluß gezogen: ,R a s chigs Behauptung ist endgültig widerlegt worden". Was kann ich, ohne beleidigend $\mathrm{zu}$ werden, dazu noch sagen? Am besten gar nichts.

Den Abschnitt III (über die Vorgänge in der Bleikammer) habe ich zu meinem Leidwesen nicht verstanden und kann daher auch nichts dagegen vorbringen. Ich sehe nur, da $\beta \mathrm{L}$ u $\mathrm{n}$ g e und $\mathrm{B}$ e $\mathrm{r} 1$ die Existenz der Nitrosulfosäure auch in verdünn- teren Schwefelsäuren nach wie vor annehmen und den Beweis dafür in der Tatsache suchen, daß auch solche Lösungen mit Kupfer oder Quecksilber Stickoxyd liefern. Ich soll das geleugnet haben. Beides ist falsch. Ich habe mich über Stickoxydbildung aus Lösungen von salpetriger Säure in schwächeren Schwefelsäuren überhaupt nicht ausgesprochen, sondern nur nachgewiesen, daß in solchen Lösungen Kupfer und Quecksilber k e i n e B l a u f ä r b u n g geben, also keine Nitrosisulfosäure erzeugen. Ich habe ferner nachgewiesen, daß der Grund des Ausbleibens der Blaufärbung nicht in der Unbeständigkeit der blauenNitrosisulfosäure bei diesen Konzentrationen zu suchen ist, also wohl nur darinliegen kann, daß die zu ihrer Entstehung nötige Nitrosulfosäure nicht da ist, sondern nur eine Lösung von salpetriger Säure in Schwefelsäure. L u n g e und Berl suchen jetzt die Erklärung für das Ausbleiben der Blaufärbung in der Bildung eines zähen Schlammes von grauem Mercurosulfat, welche die $\mathrm{W}$ a h rne $\mathrm{h} \mathbf{m}$ u $\mathrm{g}$ der Blaufärbung hindere. Hätten sie meinen Versuch wiederholt, so wie ich ihn beschreibe, mit nur einem Tropfen Quecksilber, so würden sie gesehen haben, daß von einer solchen Verdeckung gar keine Rede sein kann.

Im übrigen aber weist die Stickoxydentwicklung, welche L u n g e und B e r I mit Kupfer und Quecksilber in Lösungen von salpetriger Säure in schwächeren Schwefelsäuren beobachten, keineswegs, wie sie sagen, ,auf den gleichen Reaktionsverlauf, wie in konzentrierten Säuren hin", das soll doch heißen, auf Zwischenbildung von Nitrosisulfosäure aus vorhandener Nitrosulfosäure, sondern sie ist eine ganz allgemeine Erscheinung, die sogar in salzsaurer Lösung auftritt. Sie hat mit Zwischengliedern von Sulfosäurenatur also gar nichts zu tun. L u n g es und Berls Versuch, daraus auf Existenzmöglichkeit der Nitrosulfosäure bei den Konzentrationsverhältnissen, die in der Bleikammer herrschen, zu schließen, ist also erneut mißglückt; erst recht aber ist ihre Angabe falsch, ihre Reaktionsgleichungen seien in genügend quantitativer Annäherung als richtig befunden worden.

Ich habe mich nur noch mit ihrem Schlußsatz abzugeben, welcher lautet: ,Alle dem entgegenstehenden Behauptungen $\mathrm{R}$ a $\mathrm{s} \mathrm{c} \mathrm{h}$ i g s sind als unrichtig nachgewiesen worden; weiterhin auch die, da $\beta$ die Salpetersäureim Kam. merprozeB mit dem Schwefeldioxyd nicht reagiere."

Ich halte es für unmöglich, daß ein Unbefangener den hier gespert gedruckten Satz anders auffaßt, als rühre auch die mit ,weiterhin" gekennzeichnete Behauptung von mir her. Und doch wissen Kenner der Verhältnissse, daß das gerade Gegenteil der Fall ist. Nicht von mir, sondern von $\mathrm{L} \mathrm{u} \mathrm{n} g$ e stammt die Angabe, daß Salpetersäure im Kammerprozeß mit dem Schwefeldioxyd nicht reagiere, und ist von ihm jahrelang im Handbuch4) vertreten worden. Ich habe selbstverständlich garnichts dagegen, daß $\mathrm{Lunge}$ and Berl jetzt finden, daß $\mathrm{L}$ unge sich in diesem Punkt geirrt hat, und daß sie der chemischen Welt davon Mitteilung machen; aber ich möchte ihnen für die Zukunft doch nahe legen, 
dafür eine Form zu wählen, die es ausschließt, daß i c $\mathrm{h}$ für den Urheber der falschen Nachrichten gehalten werde.

\section{Die erste bekannte Kohlenoxyd= vergiftung.}

\author{
Von D. Binder, Wiesbaden.
}

\section{(Eingeg. den 10./8. 1907.)}

Im nachstehenden ist ohne Zweifel eine Kohlenoxydgasvergiftung beschrieben, vermutlich die erste, welche überhaupt in der Literatur vorkommt, sie hat sich im Jahre 1802 ereignet und ist in S e u m es "Spaziergang nach Syrakus" mitgeteilt. Die Ursache der Vergiftung wurde nicht erkannt, sondern dieselbe einem frisch angestrichenen Zimmer zugeschrieben. Die Stelle lantet wie folgt:

,In Cilly hätte ich auch bald meine irdische Laufbahn beschlossen : das ging so zu. Ich aB gut und viel wie gewöhnlich in der Wirtsstube und hatte bestellt, mir ein gutes Zimmer re c h t w a r m zu machen, weil es fürchterlich kalt war; denn die steiermärkischen und krainischen Winter halten sich in gutem Kredit, und der jetzige ist vorzüglich strenge. Nach der Mahlzeit ging ich auf das Zimmer, zog mich aus, stellte mich einige Minuten an den Ofen und legte mich zu Bette. Du weißt, daß ich ein gar gesunder Kerl bin und jeden Tag gut esse und jede Nacht gut schlafe. So auch hier. Aber es mochte vielleicht gegen $4 \mathrm{Uhr}$ des morgens sein, als ich durch eine furchtbare Angst geweckt wurde und den Kopf kaum heben konnte. So viel hatte ich noch Besinnung, daß ich erriet, ich schliefe in einem neugeweißten Zimmer, das man auf mein Verlangen gewaltig geheizt hatte. Als ich mich aufzurichten versuchte, um das Fenster zu öffnen, fiel ich kraftlos und dumpf auf den Pfühl zurück und verlor das Bewußtsein. Als es helle ward, erwachte ich wieder, sammelte so viel $\mathrm{Kraft}$, das Fenster zu öffnen, mich anzuziehen, in der Eile das Zimmer zu verlassen, hinunter zu taumeln und unten etwas Wein und Brot zu bestellen. Hier kam der zweite Paroxismus; ich sank am Tische hin in einem namenlosen Zustand, wie in einen lichtleeren Abgrund, wo Finsternis hinter mir zuschloß. So viel erinnere ich mich noch; ich dachte, das ist der Tod und war ruhig; sie werden mich schon gehörig begraben. Kurze Zeit darauf erwachte ich wieder unter dem entsetzlichsten Schweiße, der mich aber mit jedem Augenblick leichter ins Leben zurückbrachte. Der ganze Körper war naß, die Haare waren wie getaucht, und auf den Händen standen große Tropfen bis vorn an die Nägel. Niemand war in dem Zimmer; der Schweiß brachte mir nach der Schwere des Todes ein Gefühl unaussprechlicher Behaglichkeit. Etwas Schwindel kam zurück; nun suchte ich mich zu ermannen und nahm etwas Wein und Brot. Die Luft, dachte ich, ist die beste Arznei, und auf alle Fälle stirbt man besser in dem freien Elemente, als in der engen Kajüte. So nahm ich meinen Tornister mit großer Anstrengung auf die Schulter und ging oder wankte vielmehr fort; aber mit jedem Schritt ward ich leichter und stärker, und in einer halben Stunde fühlte ich nichts mehr ob mir gleich Hut, Kleid, Haar und Bart und das ganze Gesicht schwer bereift war, und der ganze Kerl wie schlecht verschossene Silberarbeit aussah; denn es fiel ein entsetzlich kalter Nebel. Nach zwei Stunden frühstückte ich wieder mit so gutem Appetit, als ich je getan hatte. Siehst du, lieber Freund, so hätte mich der verdammte Kalk beinahe etwas früher, als nötig ist, aus der Welt befördert. Doch vielleicht kam mir dieses auch nur so gefährlich vor, weil ich keiner solchen Phänomene von Krankheit, Ohnmacht und so weiter gewohnt bin. Etwas gewitzigt wurde ich indes dadurch für die Zukunft, und ich visitiere nun allemal erst die Wände cines geweißten Zimmers, ehe ich mich ruhig einquartiere."

Der ganze Vorgang hat die größte Ähnlichkeit mit dem Tode Z o l a s, auch dieser wollte noch das Fenster öffnen, konnte dasselbe aber nicht mehr erreichen, wie überhaupt bei allen Kohlenoxydvergiftungen. Wenn man in Betracht zieht, daß das Kohlenoxyd um das Jahr 1800 entdeckt wurde, so ist es selbstredend, daß im großen Publikum von demselben nichts bekannt war. Aber bezeichnend ist es, daß der Kalk die Ursache der Vergiftung war, es wird vermutlich auch hente noch Erkrankungen geben, die auf Vergiftungen zurückzuführen sind, und bei denen die Ursache auch wo anders gesucht wird.

\section{Über den Vorlauf des finländischen Terpentinöles (Kienöles).}

\author{
Von Osstan Aschan.
}

(Eingeg. d. 17./7. 1907.)

Zwei frühere Arbeiten 1 ) bezweckten, die Terpene zu identifizieren, welche von den in Finland heimischen beiden $P$ in $u$ s arten $P$. silvestris und $P$. a bi es erzeugt werden. In der ersten der zitierten Arbeiten wurde $u$. a. auch das Kienöl untersucht, welches als Nebenprodukt bei der trockenen Destillation der harzreichen Wurzeln abgestorbener Stämme der genannten $\mathrm{P}$ i $\mathrm{n} \mathrm{u}$ s arten in nicht unbeträchtlicher Menge ${ }^{2}$ ) abfällt und zur Bereitung von Malerfarben, Firnissen usw. gebraucht wird. Dieses Kienöl wird von den Fabrikanten durch Wasserdampfdestillation unter $\mathrm{Zu}$ gabe von Kalk gereinigt und fraktioniert aufgefangen. Je nach der relativen Farblosigkeit geht es nachher in den Handel unter verschiedenen Marken, die mit 1., 2. und 3 . bezeichnet werden. Bei der Numerierung spielt außerdem der mehr oder weniger ausgeprägte Geruch eine Rolle, welcher allen der Art erzeugten Kienölen eigen ist. Dieser Geruch ist eigentïmlich erstickend und hängt z. T. mit der gelben Farbe der Kienölfraktionen zusammen, indem die geringeren Sorten 2. und 3., welche mehr

1) Vgl. Edv. Hjelt und Ossian Aschan, Finska Vetensk.-soc. Bidrag 54, 459(1894); As c h an ibid, 46 (1896); Berl. Berichte 39 (1906).

2) Nach einer Schätzung von berufener Seite dürfte die Jahresproduktion dieses Kienöls zurzeit auf ca. $600000 \mathrm{~kg}$ zu schätzen sein. 